\title{
When the Sense of Smell Meets Emotion: Anxiety-State- Dependent Olfactory Processing and Neural Circuitry Adaptation
}

\author{
Elizabeth A. Krusemark, ${ }^{1}$ Lucas R. Novak, ${ }^{1}$ Darren R. Gitelman, ${ }^{3}$ and Wen $\mathrm{Li}^{1,2}$ \\ ${ }^{1}$ Department of Psychology and the ${ }^{2}$ Waisman Center, University of Wisconsin-Madison, Madison, Wisconsin 53706, and ${ }^{3}$ Department of Neurology, \\ Northwestern University Feinberg School of Medicine, Chicago, Illinois 60611
}

\begin{abstract}
Phylogenetically the most ancient sense, olfaction is characterized by a unique intimacy with the emotion system. However, mechanisms underlying olfaction-emotion interaction remain unclear, especially in an ever-changing environment and dynamic internal milieu. Perturbing the internal state with anxiety induction in human subjects, we interrogated emotion-state-dependent olfactory processing in a functional magnetic resonance imaging (fMRI) study. Following anxiety induction, initially neutral odors become unpleasant and take longer to detect, accompanied by augmented response to these odors in the olfactory (anterior piriform and orbitofrontal) cortices and emotion-relevant pregenual anterior cingulate cortex. In parallel, the olfactory sensory relay adapts with increased anxiety, incorporating amygdala as an integral step via strengthened (afferent or efferent) connections between amygdala and all levels of the olfactory cortical hierarchy. This anxiety-state-dependent neural circuitry thus enables cumulative infusion of limbic affective information throughout the olfactory sensory progression, thereby driving affectively charged olfactory perception. These findings could constitute an olfactory etiology model of emotional disorders, as exaggerated emotion-olfaction interaction in negative mood states turns innocuous odors aversive, fueling anxiety and depression with rising ambient sensory stress.
\end{abstract}

\section{Introduction}

The olfactory sense has a unique intimacy with emotion. Unlike other senses, olfactory neuroanatomy is intertwined, via extensive reciprocal axonal connections, with primary emotion areas including the amygdala, hippocampus, and orbitofrontal cortex (OFC) (Carmichael et al., 1994; Haberly, 2001; Gottfried, 2010). Olfactory stimulation can directly activate amygdala neurons, bypassing the primary olfactory cortex, before arriving at the secondary (association) olfactory cortex situated in the middle of the OFC. Unsurprisingly then, olfactory perception is known to be dominated by emotion. Potent affective experiences (e.g., "it smells good;" "that stinks"), as opposed to object percepts (e.g., smell of fresh linen; smell of sweaty socks), arise spontaneously in the presence of odors (Schiffman, 1974). In fact, humans are notoriously poor at odor object identification, even if they are to name smells of household objects (Lawless and Engen, 1977; Cain, 1979; de Wijk et al., 1995). Along this line, recent evidence further promotes the idea that affective valence represents the

Received May 1, 2013; revised July 1, 2013; accepted July 31, 2013.

Author contributions: W.L. designed research; E.A.K. and L.R.N. performed research; E.A.K., L.R.N., D.R.G., and W.L. analyzed data; E.A.K., D.R.G., and W.L. wrote the paper.

This work was supported by NIH grants R01MH093413 (W.L.), P3OHD03352 (W.L.), and T32MH018931 (E.A.K. and L.R.N.). We thank Jaryd Hiser for assistance with data collection.

The authors declare no competing financial interests.

Correspondence should be addressed to either Dr. Elizabeth A. Krusemark or Dr. Wen Li, Department of

Psychology, Brogden Hall, University of Wisconsin, Madison, WI 53706. E-mail: ekrusemark@wisc.edu or wenli@psych.wisc.edu.

DOI:10.1523/JNEUROSCI.1835-13.2013

Copyright $\odot 2013$ the authors $\quad 0270-6474 / 13 / 3315324-09 \$ 15.00 / 0$ dominant dimension in olfactory perception, and affective experience is the primary behavioral correlate of odorant chemical properties (Khan et al., 2007; Yeshurun and Sobel, 2010). However, despite this affective primacy in olfaction, it remains unclear how emotion and olfaction interact and converge in everyday life.

Phylogenetically the most ancient sense, olfaction has long been associated with primitive needs concerning reward, threat, and homeostasis. Human olfactory experience ties directly with the internal milieu, such that an invariant odorant elicits vastly disparate perception given different biological states, a phenomenon characterized as olfactory alliesthesia (Cabanac, 1971). A food odor thus becomes especially appetizing with decreasing metabolic energy reserve, promoting food seeking and consumption (O'Doherty et al., 2000; Gottfried et al., 2003). Intriguingly, the often affectively charged olfactory experience may resemble certain homeostatic emotions (e.g., hunger, thirst), evolutionarily engineered to sustain an organism's internal physiological equilibrium (Craig, 2003; Darwin, 1872). To some degree, this state-dependent process in olfaction also aligns with the embodied perception literature, demonstrating various perceptual alterations given different internal/bodily states (e.g., fatigue and affect) (Proffitt, 2006; Barsalou, 2008). In light of the particular relevance of olfaction in fulfilling primitive biological needs, we thus posit that olfactory perception can be largely steered by the internal state to integrate hedonic value and biological utility with basic sensory encoding (Cabanac, 1971, 1979).

To test this hypothesis, we applied a laboratory anxiety induction procedure to causally perturb the internal milieu, thereby investigating state-dependent processing of odors. We chose anx- 
A Neutral Odor

\begin{tabular}{|lc|}
\hline Pure & Mixture \\
AC 5\% & AC 5\% / AN .00025\% \\
GU 5\% & GU 5\% / EG .0005\% \\
AN 5\% & AN 5\% / AC .00025\% \\
EG 5\% & EG 5\% / GU .00025\%
\end{tabular}

Negative Odor

Mixture
AC 5\% / TM .000125\%
GU 5\% / VA. $00025 \%$

AN 5\% / TM .000025\%

EG 5\% / VA .000075\%
$A C=$ Acetophenone

$\mathrm{GU}=$ Guaiacol

AN $=$ Anisole

$E G=$ Eugenol

$\mathrm{TM}=$ Trimethylamine

$\mathrm{VA}=$ Valeric Acid

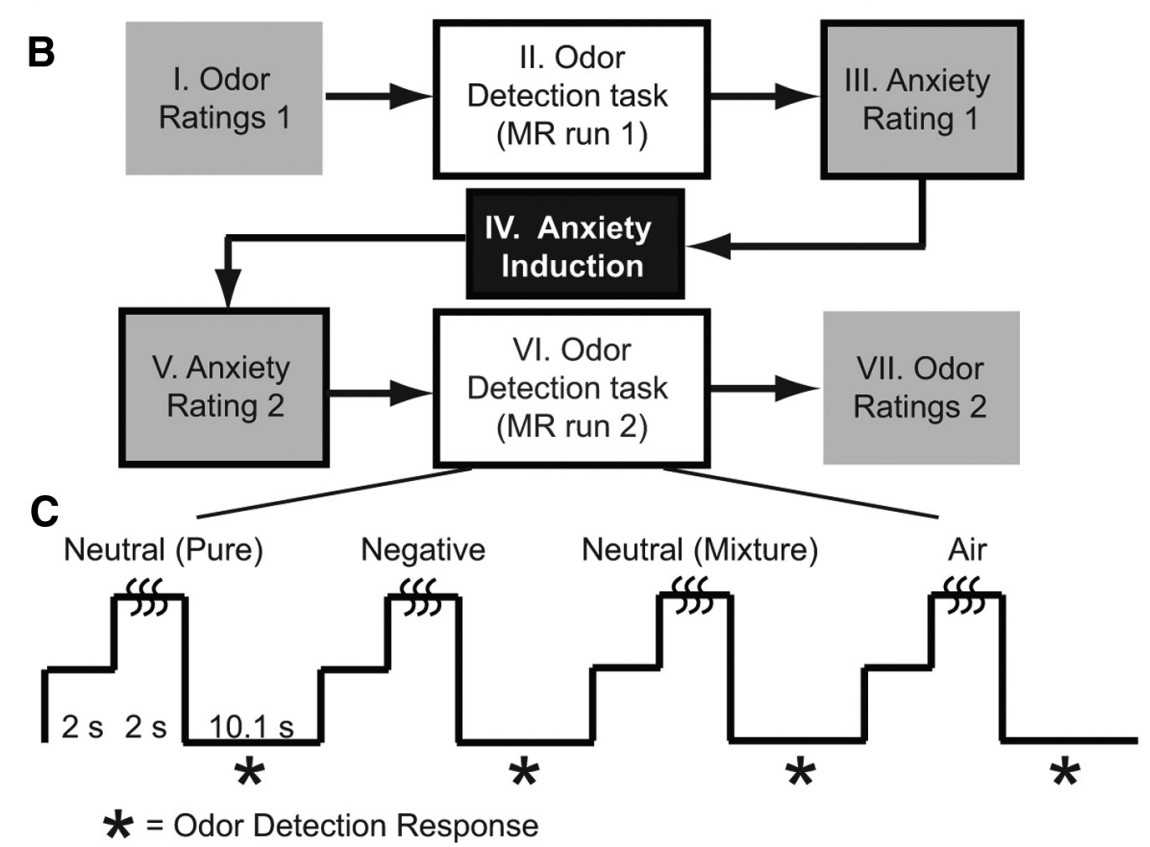

Figure 1. Study design. $\boldsymbol{A}$, Olfactory stimuli consisted of neutral odors (pure and mixture odorants) and negative odors (filler items). B, Each participant underwent a series of seven events through the study. Odor ratings (Steps I and VII) were performed runs immediately before and after a 6 -min-long anxiety induction procedure. Respiration was recorded throughout the task. outside the scanner and the others inside the scanner. C, Paradigm of the odor detection task. The task was conducted in both fMRI

confirmed that these two types of neutral odors did not differ in any major dependent variables here). The effect of anxiety on the perception of clearly negative stimuli has been inconsistent and complicated in the literature, potentially due to two opposing mechanisms at play in anxiety: one inducing negative biases and the other involving strategic, top-down inhibition (compare Williams et al., 1997; Mathews and MacLeod, 2005). As such, negative odors were included as filler items (to prevent repeated neutral odor presentation in successive trials) and to constitute a control condition. Pure room air was also included as an experimental baseline/control condition. Odors and room air were delivered using an MRI-compatible 16-channel computer-controlled olfactometer (airflow, $1.5 \mathrm{~L} / \mathrm{min}$ ) (Li et al., 2006, 2010a). Stimulus presentation and response recording were executed using Cogent software (Wellcome Department, London) implemented in MATLAB (MathWorks).

\section{Procedure}

Odor rating. At the beginning and the end of the experiment, each participant provided odor ratings using a visual analog scale (VAS) of -10 to +10 on four dimensions: valence ("extremely unpleasant" to "extremely pleasant"), intensity ("unnoticeable at all" to "strongest possible"), pungency ("not at all" to "extreme"), and familiarity ("not at all" to "extreme").

Odor detection task. Participants completed an odor detection task in pre-induction and post-induction phases in the scanner, indicating odor presence/absence by pressing one of two buttons (Fig. 1B). Each phase consisted of neutral odor (60 trials, 30/30 pure/mixture odor), negative odor (30 trials), and air condi-

iety induction to capitalize on the fact that anxiety entails both exaggerated emotion processing and a distinct constellation of strong physiological responses (Barlow, 2004; Mathews and MacLeod, 2005). Combining functional magnetic resonance imaging ( $\mathrm{fMRI}$ ) techniques with olfactory affective judgment and odor detection tasks (see Fig. 1), we specifically interrogated how emotion was infused into olfactory processing of (affectively ambiguous) neutral odors with anxiety induction. Finally, we further incorporated neural connectivity analysis (using dynamic causal modeling and psychophysiological interaction analysis; Friston et al., 1997, 2003; Gitelman et al., 2003) to unveil the neural circuitry subserving this dynamic, state-dependent process.

\section{Materials and Methods \\ Participants}

A total of 14 healthy subjects (mean age 20.83 years; range $18-28$ years; 8 males) passed our olfactory screening and provided informed consent to participate in the study, which was approved by the University of Wisconsin Madison Institutional Review Board. Two subjects with excessive motion during scans were excluded from fMRI analysis while remaining in behavioral analysis.

\section{Stimuli and stimulus presentation}

Four neutral odorants (acetophenone, guaiacol, anisole, and eugenol) and two negative odorants (trimethylamine and valeric acid) were chosen to form three odor types: neutral pure odor, neutral odor mixture, and negative odor mixture (Fig. $1 A$ ). Neutral pure and neutral mixture odors formed the main condition of neutral odors (additional analyses tions (15 trials), in a pseudo-random order with no odor type repeated over three trials in succession. Trials recurred with a stimulus onset asynchrony of $14.1 \mathrm{~s}$.

Anxiety induction. Participants underwent an anxiety induction between the two odor detection runs. Following previous work (PachecoUnguetti et al., 2010), anxiety induction involved 12 anxiety-provoking images chosen from the International Affective Picture Set (Lang et al., 2008; their images, 3010, 2683, 3051, 2811, 3000, 3015, 6550, 3068, 9419, 3012, 9433, and 3071), depicting life-threatening emotional content (e.g., mutilation, disease, or violence). During image presentation (20 s), text appeared beneath the image emphasizing the uncontrollability and unpredictability of each event (e.g., "Anybody is in danger of being abducted." "Street violence is escalating and unpredictable."). Participants' attentional engagement during the induction was reinforced by required color response to a central fixation cross (blue/yellow) immediately after each image ( $5 \mathrm{~s}$ ). Responses were monitored to ensure all participants responded accurately. Before and after anxiety induction (while remaining inside the scanner), participants provided a state anxiety rating according to the Subjective Units of Distress (Wolpe and Lazarus, 1966) on a scale from 0 (the most relaxed or calm you have ever been) to 100 (the most anxious or distressed you have ever been). This measure is commonly used in clinical settings to assess online self-reports of anxiety and discomfort (Edelman and Chambless, 1993; Muhlberger et al., 2001).

Respiratory monitoring. Respiration measurements were acquired using a BioPac MP150 system and accompanying AcqKnowledge software (BioPac Systems) with a breathing belt affixed to the subject's chest to record abdominal or thoracic contraction and expansion. Subjectspecific sniff waveforms were baseline-adjusted (based on $1000 \mathrm{~ms}$ preceding sniff onset) and then averaged across each condition before 
computing sniff inspiratory volume, peak amplitude and latency in MATLAB.

\section{Statistical analysis}

$t$ tests were conducted on anxiety ratings and odor ratings (one-tailed thresholds were applied for directional comparisons). Two-way repeated-measures ANOVAs of odor condition (Neutral odor vs Air) and anxiety (Pre-induction vs Post-induction) were applied on sniff parameters, odor detection reaction time (RT), and accuracy. RTs beyond \pm 2 standard deviations from each individual mean were excluded.

\section{Imaging acquisition and analysis}

Gradient-echo T2-weighted echoplanar images were acquired with blood-oxygen-level-dependent contrast on a 3T GE MR750 MRI scanner, using an eight channel head coil with sagittal acquisition. Imaging parameters were TR/TE $=2350 / 20 \mathrm{~ms}$; flip angle $=60^{\circ}$, field of view 240 $\mathrm{mm}$, slice thickness $2 \mathrm{~mm}$, gap $1 \mathrm{~mm}$; in-plane resolution/voxel size $1.72 \times 1.72 \mathrm{~mm}$; matrix size $128 \times 128$. A high-resolution $(1 \times 1 \times 1$ $\mathrm{mm}^{3}$ ) T1-weighted anatomical scan was also acquired. Lastly, a field map was acquired with a gradient echo sequence.

Imaging data were preprocessed using AFNI (Cox, 1996), where images were slice-time corrected, spatially realigned and coregistered with the T1, and field-map corrected. Output echoplanar images were then entered into SPM8 (http://www.fil.ion.ucl.ac.uk/spm/software/spm8/) where they were spatially normalized to a standard template, resliced to $2 \times 2 \times 2 \mathrm{~mm}^{3}$ voxels, and smoothed with a $6 \mathrm{~mm}$ full-width halfmaximum Gaussian kernel. Normalization was based on Diffeomorphic Anatomical Registration Through Exponentiated Lie algebra, a preferable approach relative to conventional normalization to achieve more precise spatial registration (Ashburner, 2007).

Next, imaging data were analyzed in SPM8 using the general linear model. Four vectors of onset times were created, corresponding to three odor types (neutral pure and neutral mixture types were pooled in statistical analysis) and the air condition, with respiratory parameters included as response modulators for each condition. These vectors were coded as delta functions and convolved with a canonical hemodynamic response function (HRF) with temporal and dispersion derivatives. Six movement-related vectors were included as regressors of no interest. A high-pass filter (cutoff, $128 \mathrm{~s}$ ) and an autoregressive model (AR1) were applied. Model estimation yielded condition-specific regression coefficients ( $\beta$ values) in a voxelwise fashion for each subject, which were later submitted to second-level, random effects group analysis for hypothesis testing.

Our neural analysis focused on a set of a priori ROIs: the olfactory sensory cortex-anterior and posterior piriform cortex (APC and PPC) and olfactory OFC (Carmichael et al., 1994; Zelano and Sobel, 2005; Gottfried, 2010); and emotion areas-amygdala and anterior cingulate cortex (ACC), especially considering their close relationship with anxiety (Rauch et al., 2003; Bishop et al., 2004; Etkin et al., 2011). Small volume of interest correction (SVC, $p<0.05$ FWE) based on the anatomical ROIs was applied to correct for multiple comparisons. The olfactory OFC ROI was based on a $6 \mathrm{~mm}$ sphere centered on the right or left putative olfactory OFC $(x, y, z: 24,36,-12 ;-22,32,-16)$ (Gottfried and Zald, 2005). Other ROIs were based on anatomical masks assembled in MRIcro (Rorden and Brett, 2000) and drawn on the mean T1 image, with reference to a human brain atlas (Mai et al., 1997). All coordinates are in Montreal Neurological Institute (MNI) space.

Psychophysiological Interaction (PPI) analysis. PPI describes the change in contribution in one brain area (seed region) and another with the experimental or psychological context (Friston et al., 1997; Gitelman et al., 2003). We chose regions arising from the conventional SPM analysis as seed regions. Volumes of interest (VOIs) were based on the ROIs above, functionally constrained at the subject level (odor $>$ air, liberally set at $p<0.5$ uncorrected to remove low-signal voxels that cover air and bone). A general linear model was estimated separately for pre-induction and post-induction sessions with three regressors: a physiological regressor for the source region (the first eigenvariate of blood-oxygenlevel-dependent-signal time series extracted from all voxels in a VOI), a psychological regressor for the effect of the experimental context (Neutral odors vs Air), and the PPI regressor (interaction between the first two regressors). Subject-level PPI regressor estimates were then entered into a paired $t$ test between pre-induction and post-induction sessions to assess the effect of anxiety on functional connectivity.

Dynamic Causal Modeling (DCM) analysis. DCM provides a Bayesian model comparison procedure to estimate effective (causal) connectivity between brain regions at the neuronal level, inferring the probable neural network given the data observed. DCM further generates parameter estimates for intrinsic connectivity (endogenous coupling between two regions) and for bilinear modulation by experimental manipulation on the intrinsic connectivity.

Guided by known anatomy and regions of interest emerging from the conventional and PPI analyses, we specified a five-region network comprising the olfactory cortical hierarchy (APC, PPC, and olfactory OFC) and two key emotion regions (amygdala and pregenual ACC/pgACC). As areas isolated in the study were all right-lateralized, in keeping with the notion of right-hemisphere dominance in olfactory processing and (negative) emotion processing (Jones-Gotman and Zatorre, 1988, 1993; Borod et al., 1998; Davidson and Irwin, 1999), we only included the right hemisphere in the model to reasonably constrain model space and complexity. We also note that this choice adhered to pertinent previous studies (Plailly et al., 2008; Dima et al., 2011).

VOIs were defined by the chosen ROIs, functionally constrained (odor $>$ air; $p<0.05$ and $p<0.25$ uncorrected for olfactory and emotion areas, respectively). We conducted model comparison using randomeffects (RFX) Bayesian model selection (BMS) in DCM10. The most likely model was then evaluated for each subject. Parameters of intrinsic connectivity generated from this analysis were subjected to one-sample $t$ tests, and modulatory parameters were assessed using twoway ANOVAs of odor condition (Neutral odor vs Air) and anxiety (Pre-induction vs Post-induction).

\section{Results}

\section{Behavioral results: anxiety shifts affective judgment and impedes detection of neutral odors}

In validation of the anxiety manipulation, state anxiety ratings indicated a significant increase in experienced anxiety from preanxiety to post-anxiety induction [mean $(\mathrm{SD})=$ Pre: 15.64 (19.06) vs Post: 40.36 (28.25)] $\left(t_{(13)}=4.99, p<0.001\right)$ (Fig. $\left.2 A\right)$. Importantly, affective judgment of odor valence indicated that neutral odors were perceived as neutral at pre-induction $[-0.59$ (1.23)] but became aversive post-induction $[-1.82(2.51)]$. The post-induction rating was significantly lower than the neutral point of zero $\left[t_{(13)}=-2.70 ; p<0.01\right.$, one-tailed] and the pre-induction baseline $\left(t_{(13)}=-1.77, p<0.05\right.$, one-tailed; Figure $\left.2 B\right)$. Though we used very weak concentrations of negative odorants to preclude stark affective contrast to neutral odors, negative mixtures were perceived as negative before the experiment $\left[-1.21(2.30) ; t_{(13)}=-1.97, p<\right.$ 0.05 , one-tailed]. Also, although the post-rating did not differ significantly from the pre-rating $(p=0.27)$, it was no longer clearly negative $\left[-0.47(1.91) ; t_{(13)}=-0.93, p=0.37\right.$; Fig. $2 B]$. We suspect this slight increase in pleasantness was related to "hedonic contrast" (Zellner et al., 2003) as neutral odors turned clearly negative.

To remove possible sequence effects or general shifts in affective judgment resulting from the mood change, we contrasted valence change in neutral versus negative odors. Our results showed significantly stronger valence change in the neutral than negative odor condition $\left(t_{(13)}=-3.03, p<0.01\right)$, indicative of a specific change in neutral (relative to negative) odors. Importantly, when we correlated this specific change in neutral odors (i.e., difference in valence change between the two conditions: $\Delta$ neutral odor valence rating $-\Delta$ negative odor valence rating) with the magnitude of induced anxiety, we observed a strong negative correlation $(r=-0.61, p<0.05$; Fig. $2 C)$. That is, a 
A

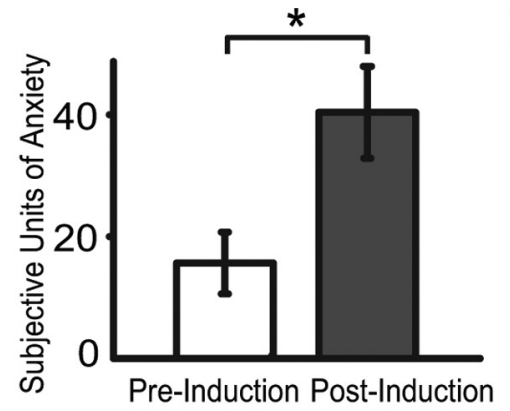

C

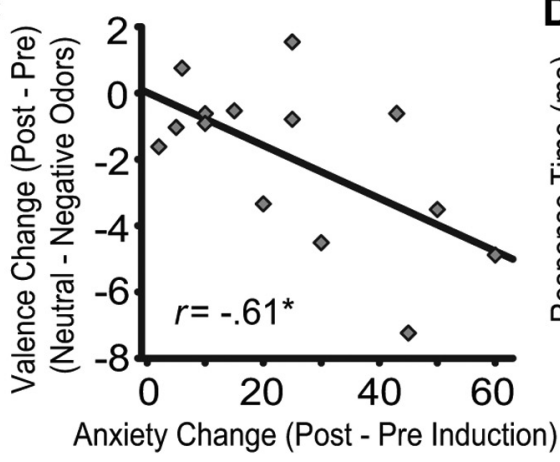

B

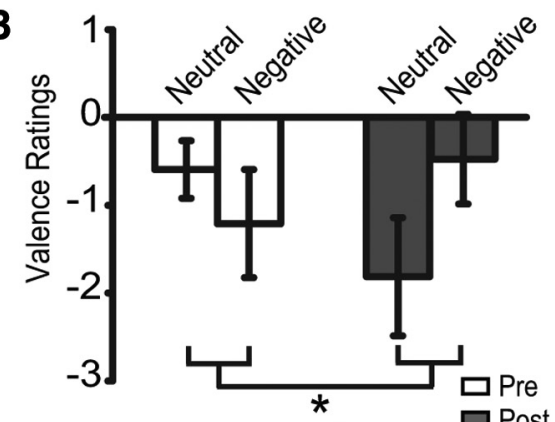

tween the magnitude of anxiety change and the sniff parameters $\left(r^{\prime} s<0.38, p^{\prime} s>\right.$ $0.22)$. We thus excluded sniff-related confounds in the reported effects.

\section{Neuroimaging results}

Because air reliably activates the olfactory system, particularly when attentive sniffs are required as in the odor detection task here (Adrian, 1942; Macrides and Chorover, 1972; Sobel et al., 1998; Fontanini et al., 2003; Zelano et al., 2005), we set the air condition as the primary experimental baseline to contrast with the neutral odor condition in the fMRI analysis. Given the complex nature of neural responses evoked by the initially negatively valenced odors as a function of anxiety, we avoided using the negative odors as the primary control condition.

\section{Anxiety amplified response to neutral odors in both olfactory and emotion systems}

Our central test of increased neural response to neutral odors (vs air) from preinduction to post-induction [Neutral odor vs Air (Post - Pre)] isolated two ROIs, representing olfactory and emotion areas, respectively. These areas were the right olfactory OFC (high-level olfactory

Figure 2. Behavioral results. $\boldsymbol{A}$, State anxiety ratings increased after induction. $\boldsymbol{B}, 0$ dor valence ratings became more negative for neutral odors after induction. $C$, Valence rating change specific to neutral odors (neutral - negative odors) correlated with magnitude of induced anxiety (post - pre). D, RTs in the odor detection task slowed down for neutral (vs air) trials after induction. Error bar indicates SEM. ${ }^{*} p>0.05$.

greater rise in anxiety was associated with a larger increase in perceived unpleasantness. To further elucidate the effect of anxiety, we performed a multiple regression analysis of anxiety change on individual rating changes for neutral and negative odors. While confirming the negative correlation between anxiety and neutral odor valence change $(s r=-0.57, p<0.05)$, we found an interesting trend-level-positive correlation between anxiety and negative odor valence change $(s r=0.49, p<0.10)$. These opposite correlations further highlight specific changes in perceived odor valence in the neutral (vs negative) condition. Finally, familiarity, intensity, and pungency ratings for the neutral odors remained largely the same between pre-induction and post-induction $\left(p^{\prime} s>0.26\right)$, thereby ruling out confounds related to olfactory learning, habituation or fatigue, which could alter these aspects of olfactory perception.

We then assayed RT and accuracy in the odor detection task during pre-induction and post-induction phases in the scanner. As is common to odor detection tasks, RTs were faster in neutral odor than no odor/air trials $\left(t_{(13)}=-6.38, p<0.001\right.$; Fig. $\left.2 D\right)$. However, this RT difference shrank from $-457 \mathrm{~ms}(334 \mathrm{~ms})$ pre-induction to $-314 \mathrm{~ms}(279 \mathrm{~ms})$ post-induction as neutral odor (vs air) responses slowed down in the post-session $\left(t_{(13)}=\right.$ 2.10, $p=0.05)$. RTs for negative odors nevertheless remained largely the same by themselves or relative to the air condition ( $\left.p^{\prime} s>0.49\right)$, which helped rule out confounds related to general sequence effects or olfactory fatigue. Accuracy analysis showed no odor or anxiety effects, independently or interactively $\left(p^{\prime} s>0.34\right)$, ruling out possible speed-accuracy tradeoffs in odor detection.

Respiratory parameters (sniff inspiratory volume, peak amplitude, and latency) were analyzed during each session, yielding no odor or anxiety effects, independently or interactively $\left(F^{\prime} s<\right.$ $\left.1.15, p^{\prime} s>0.35\right)$. Also, there was no significant correlation be- area; 26, 34, $-18 ; Z=3.10, p<0.05$ SVC; Fig. $3 A$ ) and right pgACC (high-order emotion area; $16,42,12 ; Z=3.96, p=0.01$ SVC; Fig. $3 B$ ). We also noticed a trending effect in the left APC $(-14,8,-20, Z=2.54, p=0.11$ SVC; $p=0.005$ uncorrected), showing greater response to neutral odors (vs air) after induction. Next, we examined whether these neural effects directly correlated with the magnitude of induced anxiety. To confirm the main effects and to circumvent unrealistic correlations generated by rigid correction for multiple comparisons (Vul et al., 2009), we conducted a conjunction analysis between the main contrast and a regression test of this contrast on anxiety rating change (with threshold set at $p<0.005$ uncorrected for a single contrast, resulting in a rigorous joint probability of $p<2.5 \mathrm{e}-5$ uncorrected). We observed a cluster in the right pgACC that largely overlapped with the cluster identified in the main contrast (16, 44,$14 ; Z_{\text {main effect }}=3.72, p<0.001$ uncorrected; $Z_{\text {regression }}=2.93$, $r=0.77, p=0.002$ uncorrected; Fig. $3 B$ ), showing a strong main effect that correlated positively with increasing levels of induced anxiety. Nevertheless, other a priori ROIs failed to emerge from this analysis.

\section{Anxiety strengthened connections between olfactory and} emotion systems

PPI analysis. PPI describes the change in functional coupling between one brain area (seed region) and another with varying experimental or psychological context (Friston et al., 1997; Gitelman et al., 2003). Including the two areas isolated above as seed regions, we conducted PPI analyses to examine altered functional connectivity in anxiety as possible underpinnings of the perceptual changes. The right olfactory OFC seed showed greater functional coupling with right amygdala $(20,-12,-6 ; Z=$ $3.48, p<0.05$ SVC; Fig. $3 A$ ) in response to neutral odors (vs air) at post-relative to pre-induction. The right pgACC seed, 
however, did not exhibit altered functional coupling with any ROI $(p>0.05$ uncorrected).

DCM analysis. Compared with PPI analysis, DCM analysis estimates effective (causal) connectivity between multiple brain regions, providing a more systematic and nuanced directional depiction of the neural network in question. We thus applied DCM analysis to explicate neural circuitry adaptation after anxiety induction that may underlie the interplay between olfaction and emotion.

We constructed 16 different models with bidirectional connections between low- and high-level olfaction and emotion regions, allowing experimental modulation on all paths (Fig. 4). These models varied according to four factors: the absence/presence of: (1) amygdala $\leftrightarrow$ olfactory OFC connection; (2) pgACC $\leftrightarrow$ olfactory OFC connection; (3) APC $\leftrightarrow$ amygdala connection; and (4) driving inputs to amygdala (in addition toAPC). Based on the baseline (pre-induction) data, random Bayesian model selection analysis and estimates of exceedance and expected probabilities clearly evinced Model 15 (of the 16 possible models) as the most likely model (Fig. 5A). As illustrated in Figure 5, $B$ and $C$, Model 15 included bidirectional connections between all regions except the pgACC-olfactory OFC link. We then examined intrinsic connectivity and modulation parameters for Model 15 to elucidate how anxiety influenced dynamic interactions in this neural network.

At baseline, we observed a strong feedforward projection from the amygdala to $\operatorname{pgACC}(p<0.001)$ and significant reciprocal projections between PPC and olfactory OFC (feedforward: $p<0.005$; feedback: $p<$ 0.05 ; Fig. $5 B$ ). Importantly, there were significant efferents from the amygdala to olfactory cortices (PPC and OFC; $p^{\prime}$ s < 0.05 ), laying the anatomical architecture for basic affective influences on olfaction. Following anxiety induction, amygdala efferents to PPC and olfactory OFC became particularly potent $\left(p^{\prime} s<0.0001\right.$; Fig. $5 C)$. Remarkably, two theoretically relevant but initially nonsignificant paths, $\mathrm{APC} \rightarrow \mathrm{PPC}$ and APC $\rightarrow$ amygdala, became highly important $\left(p^{\prime} s<0.0001\right)$ after induction, and these increases in intrinsic connectivity were further confirmed by paired $t$ tests, $t^{\prime} \mathrm{s}>3.06, p^{\prime} \mathrm{s}<0.01$ (Fig. 5C,D, green lines). Only one path, a feedback connection (PPC $\rightarrow$ amygdala), showed decreased connectivity in anxiety ( $p=0.05$; Fig. $5 C$, blue dashed line), but this path in itself was not significant either at pre-induction or post-induction.

Analyses of modulation parameters revealed that the presentation of neutral odors (vs air) further amplified the strength of APC $\rightarrow$ amygdala $(p<0.01)$ and amygdala $\rightarrow$ PPC $(p<0.05)$ projec-
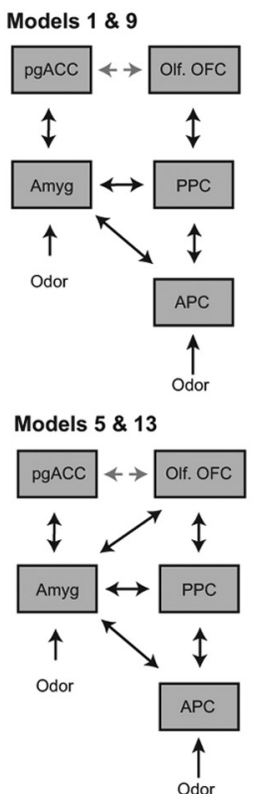

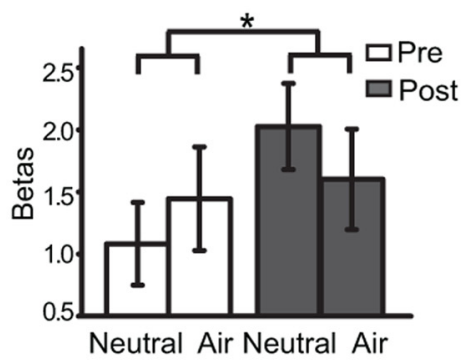

PPI (OFC seed)
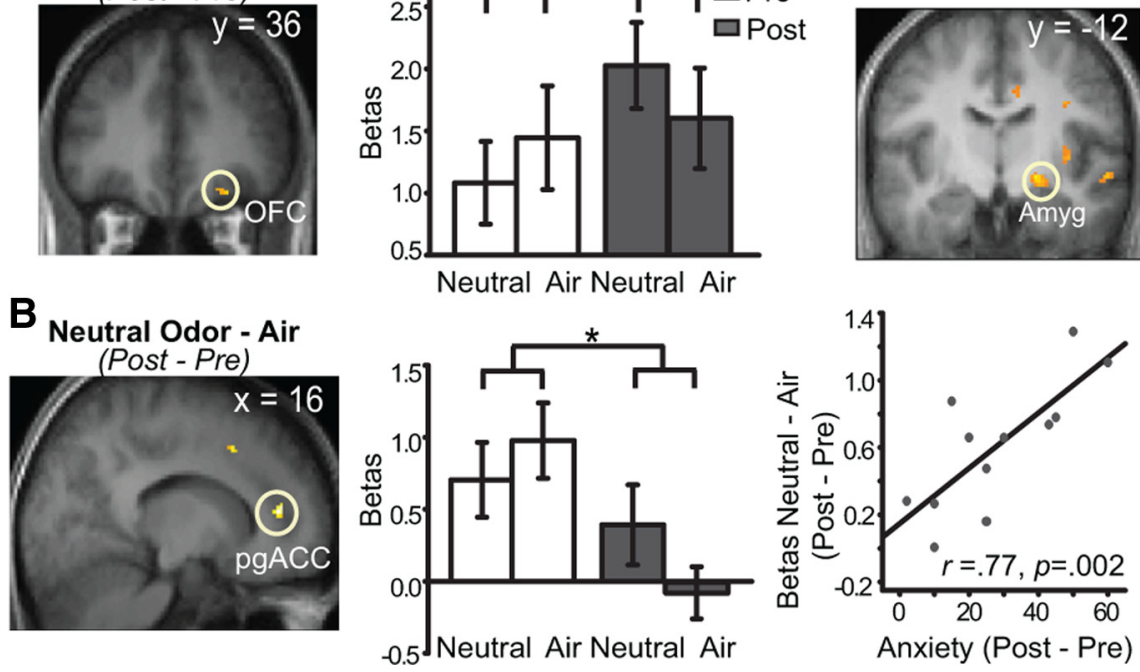

Figure 3. Conventional SPM results and PPI results. $\boldsymbol{A}$, Right olfactory OFC exhibited augmented activity and heightened functional coupling with the right amygdala (Amyg) in response to neutral odors (vs air) from pre-induction to post-induction. $\boldsymbol{B}$, Right pgACC showed response enhancement to neutral odors (vs air) from pre-induction to post-induction, correlated positively with the magnitude of induced anxiety. Activations superimposed on the group average T1 image (display threshold $p<0.005$ uncorrected). ${ }^{*} p<0.05$
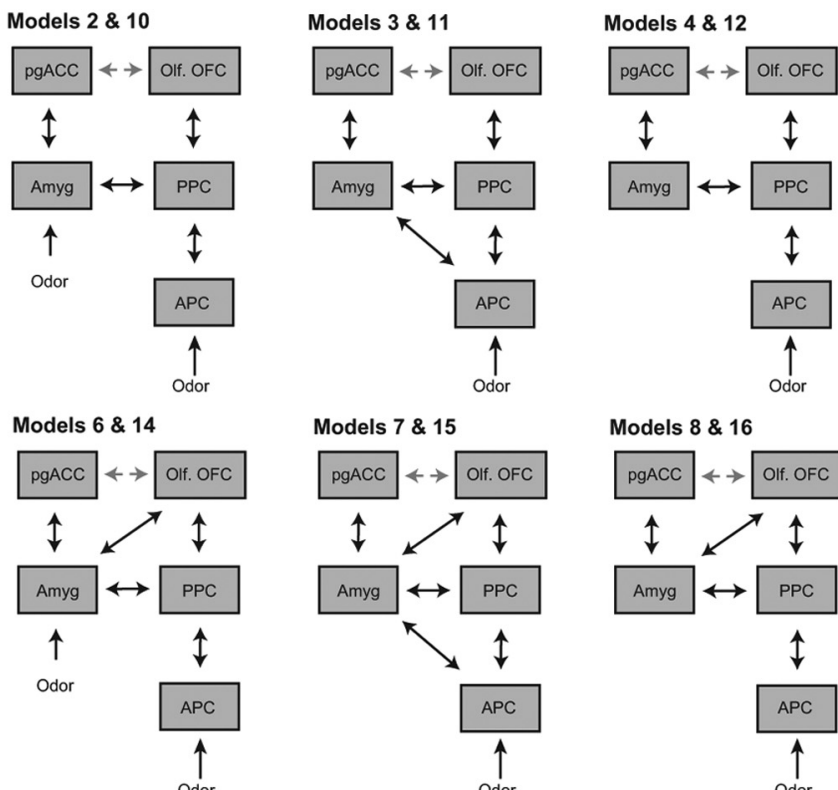

Models $8 \& 16$

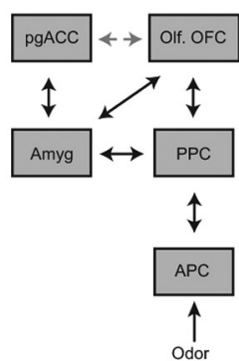

Figure 4. Model specification for DCM analyses. A five-region DCM was specified with bidirectional endogenous connections between all regions and with driving inputs of odors/air into both the APC and amygdala (models 1, 2, 5, and 6) or the APC alone (models 3, 4, 7, and 8). Sources within each model were olfactory regions (APC, PPC, and olfactory OFC) and emotion regions (amygdala/Amyg and pgACC). Models 1-8 contained a pgACC and olfactory OFC connection (illustrated as a gray line), which was absent in models 9-16. Olf. OFC, Olfactory OFC.

tions at post-induction (vs pre-induction), facilitating the crosstalk between emotion and olfactory areas in the processing of neutral odors (Fig. 5C,D, red intercepting lines). Importantly, a correlation analysis indicated that these modulatory effects (combined) were positively associated with the magnitude of induced anxiety $(r=0.60, p=$ 0.04 ), accentuating the effect of anxiety on the circuitry adaptation.

As summarized in Figure 5D, altered connections in anxiety (independently or interactively with neutral odor presentation) formed a multipath circuit of olfactory feedforward processing, comprising 


\section{A Bayesian Model Selection: RFX}

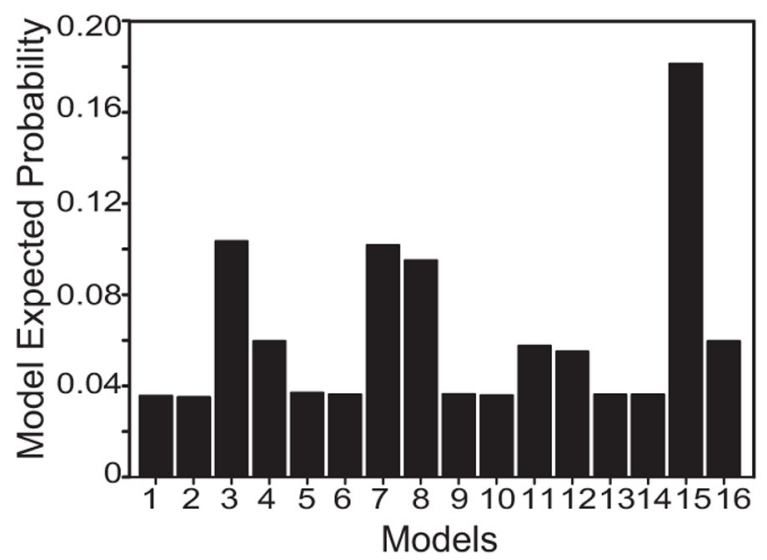

Bayesian Model Selection: RFX

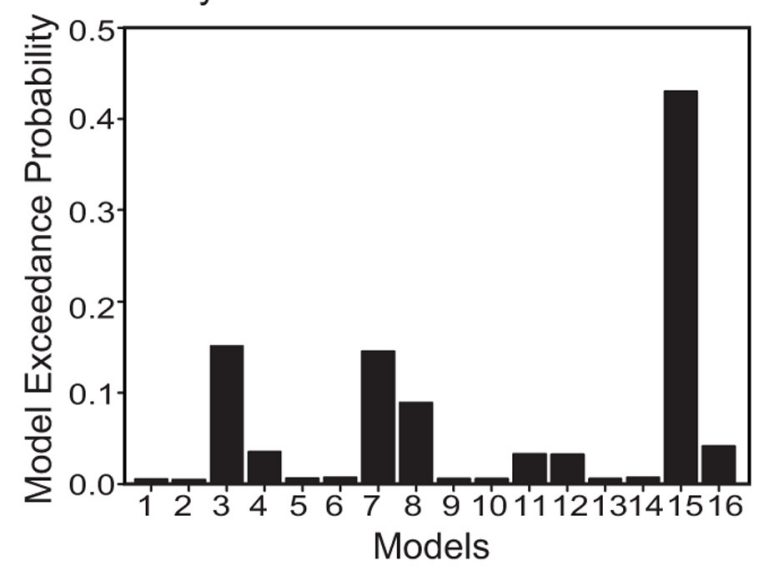

B Pre-anxiety-induction (baseline) C Post-anxiety-induction
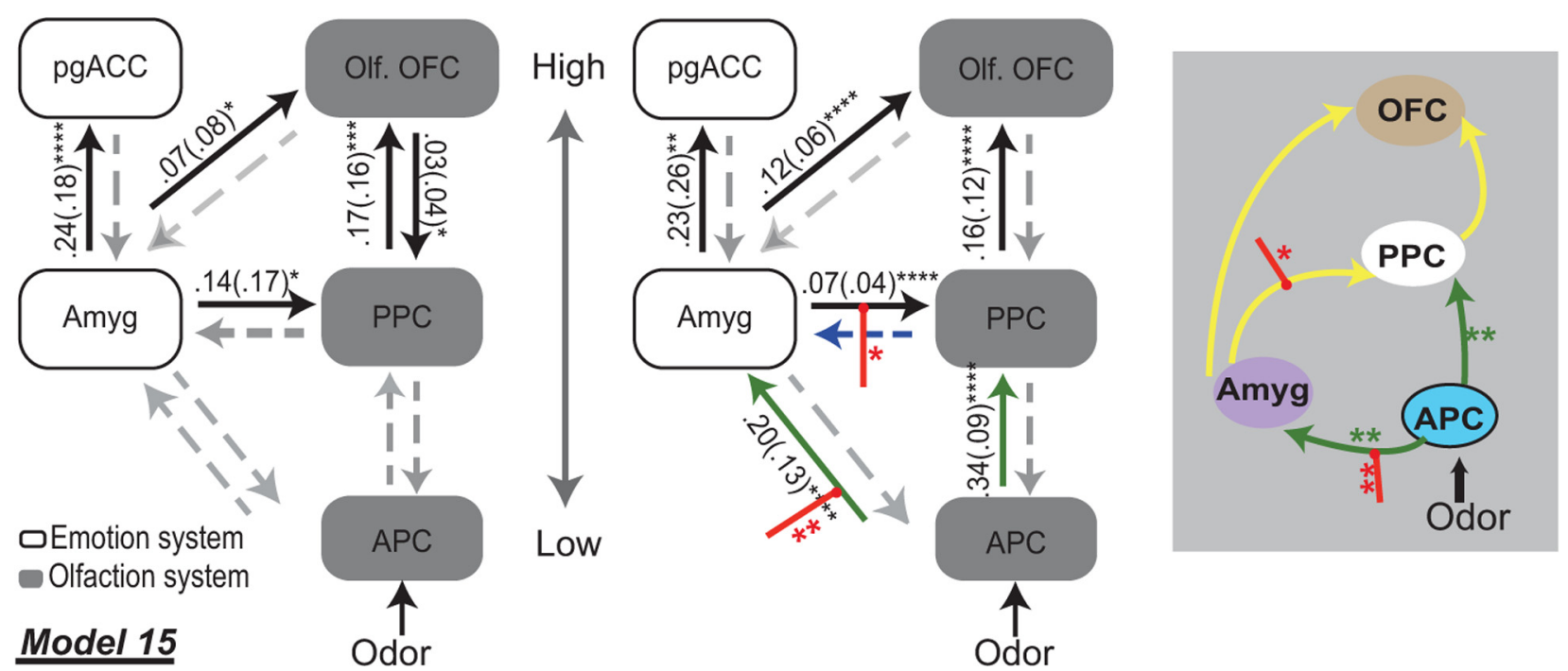

D Olfactory circuit in anxiety

Figure 5. DCM results: Neural circuitry for olfaction and emotion integration in anxiety. $\boldsymbol{A}$, Model 15 emerged as the winner with maximal expected and exceedance probabilities among the 16 models specified. $\boldsymbol{B}$, Model 15 at pre-induction baseline. Left column, Emotion areas. Right column, Olfactory areas (arranged according to the hierarchical level). Black solid lines and gray dashed lines represent significant connections (with intrinsic connectivity parameters alongside) and theoretical but nonsignificant connections, respectively. C, Model 15 at post-induction. Bolstered intrinsic connections after anxiety induction are marked as green solid lines (with intrinsic connectivity parameters alongside). The initially nonsignificant PPC $\rightarrow$ amygdala projection was further weakened by anxiety (blue dashed line). Red intercepting lines represent significant modulatory effects of neutral odor (vs air) in post-induction (vs pre-induction). $\boldsymbol{D}$, Olfactory neural circuit in anxiety. Yellow lines represent intrinsic connections initially significant, with green lines for those that became significant after anxiety induction, and red intercepting lines for modulation resulting from neutral odors at post-induction. Amyg, Amygdala; OIf. OFC, olfactory OFC. ${ }^{*} p<0.05$. ${ }^{* *} p<0.01 .{ }^{* * *} p<0.005$. ${ }^{* * *} p<0.001$.

a typical $\mathrm{APC} \rightarrow \mathrm{PPC} \rightarrow$ olfactory OFC hierarchical relay and additional routes from APC to amygdala to end in olfactory OFC directly (APC $\rightarrow$ amygdala $\rightarrow$ olfactory OFC) or via the $\mathrm{PPC}$ relay (APC $\rightarrow$ amygdala $\rightarrow \mathrm{PPC} \rightarrow$ olfactory OFC). Detailed information (intrinsic and modulatory parameters) about each specific connection is provided in Table 1.

\section{Complementary analysis of the negative odor condition}

Finally, we performed complementary analyses on neural responses to negative odors to provide further contextual insights into modified encoding of neutral odors. An analogous contrast of response change in the negative odor condition [Negative odor vs Air (Post Pre)] showed that the right $\mathrm{PPC}$ responded more strongly to negative odors after induction $(18,4,-18, Z=3.47, p<0.05$ SVC), and the right APC also exhibited a trending effect $(16,8,-20, Z=2.86$; $p<0.005$ uncorrected). Nevertheless, the higher-level areas did not exhibit any effects surviving the statistical threshold ( $p<0.05$ SVC): no voxel in olfactory OFC survived the threshold of $p<0.01$ uncor- rected, and in pgACC, there was a similar but weaker effect to the one observed in the neutral odor condition [a single voxel $(16,44,12)$ at $p<0.001$ uncorrected, $Z=3.04 ; 2$ voxels at $p<0.005$ uncorrected). A similar set of analyses on DCM connectivity parameters was also conducted, which failed to reveal any significant effects in the negative odor condition ( $p^{\prime} s>0.16$ ). Together, these results suggest that, in the absence of heightened interaction between the amygdala and the olfactory cortex in the negative condition, the influence of anxiety in negative odor processing was restricted to low-level sensory processing, failing to reach the olfactory OFC (and perhaps pgACC) to directly impact odor perception.

\section{Discussion}

Following experimental anxiety induction, prototypical neutral odors became negatively valenced and required more time to detect. This shift in affective perception was closely correlated with the magnitude of induced anxiety, further underscoring a 
Table 1. Parameter estimates of intrinsic and modulatory parameters for Model 15

\begin{tabular}{|c|c|c|c|c|c|}
\hline \multirow[b]{2}{*}{ Pathway } & \multicolumn{2}{|l|}{ Intrinsic connections } & \multicolumn{3}{|l|}{ Modulatory parameters } \\
\hline & Mean (SD) Pre/Post & T, p (Post/Pre) & Neutral (Pre/Post)/Mean (SD) & Air (Pre/Post)/Mean (SD) & Anxiety $\times 0 \operatorname{dor}(F, p)$ \\
\hline $\mathrm{APC} \rightarrow$ Amyg & $-0.09(0.23) / 0.20(0.13)^{* * * *}$ & $3.33,0.007^{* *}$ & $0.002(0.061) / 0.106(0.076)$ & $0.063(0.073) / 0.080(0.085)$ & $9.52,0.01^{* *}$ \\
\hline $\mathrm{APC} \rightarrow \mathrm{PPC}$ & $0.007(0.33) / 0.34(0.09)^{* * * *}$ & $3.06,0.01^{*}$ & $0.044(0.059) / 0.070(0.052)$ & $0.058(0.072) / 0.045(0.056)$ & $1.56,0.24$ \\
\hline $\mathrm{PPC} \rightarrow$ Amyg & $0.05(0.10) /-0.04(0.13)$ & $-2.28,0.04^{*}$ & $0.004(0.007) / 0.008(0.009)$ & $0.001(0.010) / 0.005(0.008)$ & $0.003,0.96$ \\
\hline $\mathrm{PPC} \rightarrow \mathrm{APC}$ & $-0.001(0.07) / 0.03(0.11)$ & $0.88,0.39$ & $0.002(0.003) / 0.001(0.002)$ & $-0.001(0.007) / 0.001(0.001)$ & $1.33,0.27$ \\
\hline $\mathrm{PPC} \rightarrow$ 0lf. OFC & $0.17(0.16)^{* * *} / 0.16(0.12)^{* * * *}$ & $-0.16,0.88$ & $0.003(0.024) / 0.018(0.019)$ & $0.005(0.016) / 0.003(0.005)$ & $3.03,0.11$ \\
\hline Amyg $\rightarrow$ APC & $-0.01(0.09) / 0.03(0.05) \dagger$ & $1.12,0.29$ & $-0.002(0.009) / 0.001(0.001)$ & $-0.001(0.006) / 0.001(0.001)$ & $0.79,0.39$ \\
\hline Amyg $\rightarrow$ PPC & $0.14(0.17)^{*} / 0.07(0.04)^{* * * *}$ & $-1.69,0.12$ & $-0.001(0.009) / 0.005(0.006)$ & $0.003(0.005) / 0.003(0.004)$ & $4.78,0.05^{*}$ \\
\hline Amyg $\rightarrow$ Olf. OFC & $0.07(0.08)^{*} / 0.12(0.06)^{* * * *}$ & $1.73,0.11$ & $0.002(0.021) / 0.019(0.020)$ & $0.002(0.017) / 0.004(0.007)$ & $1.49,0.25$ \\
\hline Amyg $\rightarrow$ pgACC & $0.24(0.18)^{* * * *} / 0.23(0.26)^{* *}$ & $-0.72,0.94$ & $0.006(0.009) / 0.018(0.019)$ & $0.010(0.017) / 0.018(0.024)$ & $0.13,0.72$ \\
\hline Olf. OFC $\rightarrow$ Amyg & $0.004(0.03) /-0.02(0.03) \dagger$ & $-1.79,0.10 \dagger$ & $0.002(0.006) / 0.004(0.001)$ & $-0.001(0.004) / 0.001(0.001)$ & $1.42,0.36$ \\
\hline Olf. OFC $\rightarrow$ PPC & $0.03(0.04)^{*} / 0.03(0.06) \dagger$ & $0.21,0.84$ & $-0.001(0.003) / 0.001(0.001)$ & $0.001(0.002) / 0.001(0.001)$ & $0.58,0.46$ \\
\hline $\mathrm{pgACC} \rightarrow$ Amyg & $0.02(0.04) /-0.002(0.04)$ & $-1.29,0.22$ & $0.001(0.002) / 0.001(0.001)$ & $0.002(0.006) / 0.001(0.001)$ & $0.35,0.57$ \\
\hline
\end{tabular}

${ }^{*} p<0.05 ;{ }^{* *} p<0.01 ;{ }^{* * *} p<0.005 ;{ }^{* * * *} p<0.001 ;+p<0.10$; two-tailed. Amyg, Amygdala; APC, anterior piriform; Olf. OFC, olfactory OFC; $\mathrm{pgACC}$, pregenual ACC; PPC, posterior piriform.

causal role of anxiety in infusing emotion into olfactory processing. In parallel, pgACC and olfactory OFC exhibited response enhancement to neutral odors post-induction, implicating both affective and sensory systems in anxiety-state-dependent olfactory processing. Connectivity analyses delineated neural circuitry adaptation after anxiety induction, highlighting intensified interaction between emotion and olfaction networks via strengthened feedforward and feedback connections. This rewired, highly integrative neural circuitry thus constitutes the neural mechanism underlying emotion-olfaction synthesis. An important implication is that such dynamic changes could not only mediate anxietyrelated olfactory alliesthesia (shown here) but also would account for emotion-laden olfaction in general (Yeshurun and Sobel, 2010).

Sensory processing is the entry point where an organism interacts with the external world. Evidence accrues that this process concerns not only the physical/chemical properties of the input (e.g., stimulus quality and intensity) but also its affective value (Young, 1959; Craig, 2003; Barrett and Bar, 2009). Indeed, our intrinsic connectivity mapping reveals unification between emotion and olfaction networks via robust amygdala efferents to intermediate and high-level olfactory cortices (i.e., PPC and olfactory OFC), beyond basic feedforward projections within each network. Given the role of PPC in quality encoding and olfactory OFC in hedonic evaluation and conscious experience of odors (Gottfried and Zald, 2005; Li et al., 2006, 2010a,b), direct amygdala influxes to these regions would enable emotion infusion into basic olfactory processing, thereby underpinning affectively charged odor percepts and experiences in everyday life.

Moreover, sensory (in particular, olfactory) perception occurs not in a vacuum but rather in a rich context of internal physiological and psychological states (Cabanac, 1979; Barsalou, 2008; Barrett and Bar, 2009). By experimentally inducing anxiety, known to evoke drastic psychological and physiological changes, we demonstrated olfactory perceptual alterations in the form of varied odor valence rating and detection speed. These anxietyrelated changes add to previous reports of olfactory perceptual changes in negative affective states (Chen and Dalton, 2005; Pollatos et al., 2007) and emotional disorders (Lombion-Pouthier et al., 2006; Atanasova et al., 2010; Naudin et al., 2012), in support of mood-state-related olfactory alliesthesia. In parallel to these behavioral changes, we also observed anxiety-induced neural plasticity in enhanced local brain response (in the olfactory OFC) and heightened connectivity between olfactory cortices and the amygdala (i.e., APC $\rightarrow$ amygdala and amygdala $\rightarrow$ PPC and olfactory OFC).

The theoretically relevant APC $\rightarrow$ amygdala path was (statistically) insignificant at the baseline and became prominent only after anxiety induction. This connection was particularly intensified by neutral odors post-anxiety induction. As a result, a new hierarchical feedforward route emerges (in addition to the typical $\mathrm{APC} \rightarrow \mathrm{PPC} \rightarrow$ olfactory OFC relay), through which some APC output first arrives in the amygdala before reaching higher-level olfactory cortices (Fig. 5C). As the amygdala $\rightarrow$ PPC projection plays an important role in integrative processing of odors (Small et al., 2013), this circuitry reorganization in anxiety promotes emotion-olfaction integration by incorporating amygdala as an integral step in olfactory sensory progression. Consequently, negatively charged perception of neutral odors evolves throughout the olfactory relay to culminate in the olfactory OFC. In keeping with that, the olfactory OFC exhibited augmented activity and heightened functional coupling with amygdala in response to neutral odors post-anxiety induction. Notably, this extra APC $\rightarrow$ amygdala step during neutral odor processing in anxiety may come at a response speed cost, as indicated by delayed detection of neutral odors (vs air) at post-induction.

The APC, a low-level olfactory cortex, is known to represent chemical properties of odor molecules and to maintain external signal fidelity (Gottfried, 2010). However, human fMRI research also indicates that this structure can exhibit response adaptation to psychological influences such as attention (Zelano et al., 2005) and expectation (Zelano et al., 2011). Of particular relevance here, APC supports predictive codes of upcoming olfactory stimuli and maintains neural representation of expected odors despite the actual presentation of a disparate odor (Zelano et al., 2011). Characterized by negative anticipation of the world and the future (Beck, 1976; Mathews and MacLeod, 2005), anxiety could negatively shift predictive codes in the APC to predominantly represent negative olfactory cues even when actual odor stimuli are affectively neutral. Consistently, our data demonstrated potentiated APC response to neutral odors (vs air) at postinduction (at a slightly less stringent threshold of $p=0.005$ uncorrected). In sum, biologically oriented olfactory processing may occur from the beginning of the cortical relay (in the APC), steering downstream analysis to convey motivational and affective information grounded in a given internal state (e.g., anxious state). Potentially unique to the olfactory system, this emotion encoding initiated in the sensory cortex presents an interesting alternative to prevalent accounts of emotion processing centered on the amygdala (LeDoux, 1995; Tamietto and de Gelder, 2010).

The pgACC exhibited heightened response to neutral odors in anxiety, which was parametrically coupled with the actual magnitude of induced anxiety. Nevertheless, connectivity between this area and the rest of the network did not adapt following anxiety induction. We surmise that this pgACC response en- 
hancement is likely to represent high-order, cognitive appraisal/ interpretation of the newly instilled negativity in the odors (Kalisch et al., 2006; Rushworth et al., 2007). Anxiety has been associated with interpretational biases (Mathews and MacLeod, 2005), particularly for ambiguous/neutral cues (Eysenck et al., 1991; Clark and Wells, 1995). Current findings may provide an olfactory model of this anomaly specifically and of anxiety pathology in general. That is, anxiety negatively shifts olfactory predictive coding and intensifies emotion-olfaction interaction, promoting negative affective infusion into basic olfactory perception. The rostral ACC then registers the negative value and assigns negative interpretations to otherwise innocuous smells, conferring sensory displeasure/stress to fuel and perpetuate anxiety.

We note that repeated odor exposure may lead to olfactory fatigue, adaptation, or perceptual learning. Additionally, general mood change in consequence to anxiety induction can cause general shifts in affective experience and responding. However, the fact that changes in valence and detection speed were salient in neutral, but not negative, odors helped rule out these general sequence effects as potential confounds in the current findings. Also, the close associations between induced anxiety and behavioral and neural effects in the neutral condition further accentuate the specific impact of anxiety in updating neutral odor encoding. Furthermore, perceived intensity, pungency, and familiarity of neutral odors remained invariant, indicating that olfactory fatigue or learning, if it occurred, was negligible. Finally, possible random changes in neutral odor valence ratings are ruled out by the fact that this effect was in the predicted direction (parametrically coupled with the level of induced anxiety), which further coincided with odor detection RT change and dovetailed with the neural effects. Nevertheless, to the extent that these analyses largely excluded the possible confounds, future investigation incorporating a full control group with non-anxiety (neutral) induction would provide additional validation of the findings.

A system minimally equipped with a small volume of dedicated brain structures and a simple hierarchical architecture, the human olfactory sense nonetheless needs to decipher a rich, highdimensional world of smell. Olfactory processing is thus evolutionarily pressed to operate in a highly efficient fashion. Preset toward fluctuations in the biological landscape, albeit compromising encoding in other domains (e.g., odor quality), olfaction interacts with emotion from the bottom of the cortical hierarchy via dense reciprocal connections. As such, the olfactory system drastically cuts down neural computation load and manages to accomplish its daunting mission on a daily basis. In this sense, human olfactory processing is affectively charged long before an odorous molecule makes contact with the nose.

\section{References}

Adrian ED (1942) Olfactory reactions in the brain of the hedgehog. J Physiol 100:459-473. Medline

Ashburner J (2007) A fast diffeomorphic image registration algorithm. Neuroimage 38:95-113. CrossRef Medline

Atanasova B, El-Hage W, Chabanet C, Gaillard P, Belzung C, Camus V (2010) Olfactory anhedonia and negative olfactory alliesthesia in depressed patients. Psychiatry Res 176:190-196. CrossRef Medline

Barlow DH (2004) Anxiety and its disorders. New York: Guilford.

Barrett LF, Bar M (2009) See it with feeling: affective predictions during object perception. Philos Trans R Soc B 364:1325-1334. CrossRef Medline

Barsalou LW (2008) Grounded cognition. Annu Rev Psychol 59:617-645. CrossRef Medline

Beck AT (1976) Cognitive therapy and the emotional disorders. New York: International Universities.
Bishop S, Duncan J, Brett M, Lawrence AD (2004) Prefrontal cortical function and anxiety: controlling attention to threat-related stimuli. Nat Neurosci 7:184-188. CrossRef Medline

Borod JC, Cicero BA, Obler LK, Welkowitz J, Erhan HM, Santschi C, Grunwald IS, Agosti RM, Whalen JR (1998) Right hemisphere emotional perception: evidence across multiple channels. Neuropsychology 12:446-458. CrossRef Medline

Cabanac M (1971) Physiological role of pleasure. Science 173:1103-1107. CrossRef Medline

Cabanac M (1979) Sensory pleasure. Q Rev Biol 54:1-29. CrossRef Medline Cain WS (1979) To know with the nose: keys to odor identification. Science 203:467-470. CrossRef Medline

Carmichael ST, Clugnet MC, Price JL (1994) Central olfactory connections in the macaque monkey. J Comp Neurol 346:403-434. CrossRef Medline

Chen D, Dalton P (2005) The effect of emotion and personality on olfactory perception. Chem Senses 30:345-351. CrossRef Medline

Clark DM, Wells A (1995) Social phobia, diagnosis, assessment and treatment (Heimberg R, Leibowitz M, Hope DA, Schneier FR, eds). New York: Guilford.

Cox RW (1996) AFNI: software for analysis and visualization of functional magnetic resonance images. Comput Biomed Res 29:162-173. CrossRef Medline

Craig AD (2003) Interoception: the sense of the physiological condition of the body. Curr Opin Neurobiol 13:500-505. CrossRef Medline

Darwin C (1872) The expression of emotion in man and animals. New York: Philosophical Library.

Davidson RJ, Irwin W (1999) The functional neuroanatomy of emotion and affective style. Trends Cogn Sci 3:11-21. CrossRef Medline

de Wijk R, Schab FR, Cain WS (1995) Odor identification. In: Memory for odors (Schab FR, Crowder RG, eds.). Mahwah, NJ: Lawrence Erlbaum.

Dima D, Stephan KE, Roiser JP, Friston KJ, Frangou S (2011) Effective connectivity during processing of facial affect: evidence for multiple pathways. J Neurosci 31:14378-14385. CrossRef Medline

Edelman RE, Chambless DL (1993) Compliance during sessions and homework in exposure-based treatment of agoraphobia. Behav Res Ther 31: 767-773. CrossRef Medline

Etkin A, Egner T, Kalisch R (2011) Emotional processing in anterior cingulate and medial prefrontal cortex. Trends Cogn Sci 15:85-93. CrossRef Medline

Eysenck MW, Mogg K, May J, Richards A, Mathews A (1991) Bias in interpretation of ambiguous sentences related to threat in anxiety. J Abnorm Psychol 100:144-150. CrossRef Medline

Fontanini A, Spano P, Bower JM (2003) Ketamine-xylazine-induced slow $(<15 \mathrm{~Hz})$ oscillations in the rat piriform (olfactory) cortex are functionally correlated with respiration. J Neurosci 23:7993-8001. Medline

Friston KJ, Buechel C, Fink GR, Morris J, Rolls E, Dolan RJ (1997) Psychophysiological and modulatory interactions in neuroimaging. Neuroimage 6:218-229. CrossRef Medline

Friston KJ, Harrison L, Penny W (2003) Dynamic causal modeling. Neuroimage 19:1273-1302. CrossRef Medline

Gitelman DR, Penny WD, Ashburner J, Friston KJ (2003) Modeling regional and psychophysiologic interactions in fMRI: the importance of hemodynamic deconvolution. Neuroimage 19:200-207. CrossRef Medline

Gottfried JA (2010) Central mechanisms of odour object perception. Nat Rev Neurosci 11:628-641. CrossRef Medline

Gottfried JA, Zald DH (2005) On the scent of human olfactory orbitofrontal cortex: meta-analysis and comparison to non-human primates. Brain Res Brain Res Rev 50:287-304. CrossRef Medline

Gottfried JA, O'Doherty J, Dolan RJ (2003) Encoding predictive reward value in human amygdala and orbitofrontal cortex. Science 301:11041107. CrossRef Medline

Haberly LB (2001) Parallel-distributed processing in olfactory cortex: new insights from morphological and physiological analysis of neuronal circuitry. Chem Senses 26:551-576. CrossRef Medline

Jones-Gotman M, Zatorre RJ (1988) Olfactory identification deficits in patients with focal cerebral excision. Neuropsychologia 26:387-400. CrossRef Medline

Jones-Gotman M, Zatorre RJ (1993) Odor recognition memory in humans: role of right temporal and orbitofrontal regions. Brain Cogn 22:182-198. CrossRef Medline

Kalisch R, Wiech K, Critchley HD, Dolan RJ (2006) Levels of appraisal: a 
medial prefrontal role in high-level appraisal of emotional material. Neuroimage 30:1458-1466. CrossRef Medline

Khan RM, Luk CH, Flinker A, Aggarwal A, Lapid H, Haddad R, Sobel N (2007) Predicting odor pleasantness from odorant structure: pleasantness as a reflection of the physical world. J Neurosci 27:10015-10023. CrossRef Medline

Lang PJ, Bradley MM, Cuthbert BN (2008) International affective picture system (IAPS): affective ratings of pictures and instruction manual Technical Report A-8. Gainesville, FL: University of Florida.

Lawless H, Engen T (1977) Associations to odors: interference, mneumonics, and verbal labeling. J Exp Psychol Hum Learn 3:52-59. CrossRef Medline

LeDoux JE (1995) Emotion: clues from the brain. Annu Rev Psychol 46: 209-235. CrossRef Medline

Li W, Luxenberg E, Parrish T, Gottfried JA (2006) Learning to smell the roses: experience-dependent neural plasticity in human piriform and orbitofrontal cortices. Neuron 52:1097-1108. CrossRef Medline

Li W, Lopez L, Osher J, Howard JD, Parrish TB, Gottfried JA (2010a) Right orbitofrontal cortex meditates conscious olfactory perception. Psychol Sci 21:1454-1463. CrossRef Medline

Li W, Howard JD, Gottfried JA (2010b) Disruption of odour quality coding in piriform cortex mediates olfactory deficits in Alzheimer's disease. Brain 133:2714-2726. CrossRef Medline

Lombion-Pouthier S, Vandel P, Nezelof S, Haffen E, Millot JL (2006) Odor perception in patients with mood disorders. J Affect Disord 90:187-191. CrossRef Medline

Macrides F, Chorover SL (1972) Olfactory bulb units: activity correlated with inhalation cycles and odor quality. Science 175:84-87. CrossRef Medline

Mai J, Assheuer J, Paxinos G (1997) Atlas of the human brain. London: Academic.

Mathews A, MacLeod C (2005) Cognitive vulnerability to emotional disorders. Annu Rev Clin Psychol 1:167-195. CrossRef Medline

Mühlberger A, Herrmann MJ, Wiedemann G, Ellgring H, Pauli P (2001) Repeated exposure of flight phobics to flights in virtual reality. Behav Res Ther 39:1033-1050. CrossRef Medline

Naudin M, El-Hage W, Gomes M, Gaillard P, Belzung C, Atanasova B (2012) State and trait olfactory markers of major depression. PLoS One 7: e46938. CrossRef Medline

O’Doherty J, Rolls ET, Francis S, Bowtell R, McGlone F, Kobal G, Renner B, Ahne G (2000) Sensory-specific satiety-related olfactory activation of the human orbitofrontal cortex. Neuroreport 11:893-897. CrossRef Medline

Pacheco-Unguetti AP, Acosta A, Callejas A, Lupiáñez J (2010) Attention and anxiety: different attentional functioning under state and trait anxiety. Psychol Sci 21:298-304. CrossRef Medline

Plailly J, Howard JD, Gitelman DR, Gottfried JA (2008) Attention to odor modulates thalamocortical connectivity in the human brain. J Neurosci 28:5257-5267. CrossRef Medline
Pollatos O, Kopietz R, Linn J, Albrecht J, Sakar V, Anzinger A, Schandry R, Wiesmann M (2007) Emotional stimulation alters olfactory sensitivity and odor judgment. Chem Senses 32:583-589. CrossRef Medline

Proffitt DR (2006) Distance perception. Curr Dir Psychol Sci 15:131-135. CrossRef

Rauch SL, Shin LM, Wright CI (2003) Neuroimaging studies of amygdala function in anxiety disorders. Ann N Y Acad Sci 985:389-410. CrossRef Medline

Rorden C, Brett M (2000) Stereotaxic display of brain lesions. Behav Neurol 12:191-200. Medline

Rushworth MF, Buckley MJ, Behrens TE, Walton ME, Bannerman DM (2007) Functional organization of the medial frontal cortex. Curr Opin Neurobiol 17:220-227. CrossRef Medline

Schiffman SS (1974) Physicochemical correlates of olfactory quality. Science 185:112-117. CrossRef Medline

Small DM, Veldhuizen MG, Green B (2013) Sensory neuroscience: taste responses in primary olfactory cortex. Curr Biol 23:R157-R159. CrossRef Medline

Sobel N, Prabhakaran V, Desmond JE, Glover GH, Goode RL, Sullivan EV, Gabrieli JD (1998) Sniffing and smelling: separate subsystems in the human olfactory cortex. Nature 392:282-286. CrossRef Medline

Tamietto M, de Gelder B (2010) Neural bases of the non-conscious perception of emotional signals. Nat Rev Neurosci 11:697-709. CrossRef Medline

Vul E, Harris C, Winkielman P, Pashler H (2009) Puzzlingly high correlations in fMRI studies of emotion, personality, and social cognition. Perspect Psychol Sci 4:274-290. CrossRef

Williams JM, Watts FN, MacLeod C, Mathews A (1997) Cognitive psychology and emotional disorders, Ed 2. Chichester, NY: Wiley.

Wolpe J, Lazarus AA (1966) Behavior therapy and techniques. New York: Pergamon.

Yeshurun Y, Sobel N (2010) An odor is not worth a thousand words: from multidimensional odors to unidimensional odor objects. Annu Rev Psychol 61:219-241. CrossRef Medline

Young PT (1959) The role of affective processes in learning and motivation. Psychol Rev 66:104-125. CrossRef Medline

Zelano C, Sobel N (2005) Humans as an animal model for systems-level organization of olfaction. Neuron 48:431-454. CrossRef Medline

Zelano C, Bensafi M, Porter J, Mainland J, Johnson B, Bremner E, Telles C, Khan R, Sobel N (2005) Attentional modulation in human primary olfactory cortex. Nat Neurosci 8:114-120. CrossRef Medline

Zelano C, Mohanty A, Gottfried JA (2011) Olfactory predictive codes and stimulus templates in piriform cortex. Neuron 72:178-187. CrossRef Medline

Zellner DA, Rohm EA, Bassetti TL, Parker S (2003) Compared to what? Effects of categorization on hedonic contrast. Psychon Bull Rev 10:468473. CrossRef Medline 